\title{
fisicoquímica del sistema refractario-clínker en los hornos de cemento
}

\author{
FRANCISCO SORIA SANTAMARIA \\ Licenciado en Ciencias, Jefe de la Sección de Fabricación de Cemento del I.E.T. c.c \\ Conferencia pronunciada en Barcelona, el pasade \\ mes de noviembre, con motivo de la it Reunín \\ Téenica de la Secoín de Refractarios de la Sociedad \\ Española de Cerámios.
}

\section{Introducción}

La industria del cemento, como todas aquellas que su producto base se obtiene a altas temperaturas, dedica un interés especial al sistema de cocción.

La conservación de los hornos tiene una gran importancia, desde el punto de vista económico, por el elevado coste de su instalación, exigiendo mucha atención por dos razones: su gran tonelaje y las elevadas temperaturas creadas en su interior,

Como ya saben, para preservar su cubierta metálica, se revisten interiormente de un forro refractario, cuya misión principal es encauzar el fuego-evitando pérdidas caloríficas notables y elevando, en consecuencia, el rendimiento térmico-y dar origen, en segundo término, a las reacciones del crudo de cemento que, como sabemos, sólo tienen lugar a altas temperaturas.

En los últimos años han crecido las exigencias de trabajo de los materiales refractarios con aparición de los supercementos, materiales más altos en cal y que exigen, en consecuencia, mayores temperaturas para llegar a su sinterización perfecta.

Por este motivo, siendo ya un concepto de valor apreciable el considerar el coste de fabricación, los fabricantes de cemento, como usuarios de los materiales refractarios, están interesados en estudiar y conocer las condiciones que acortan la vida de estos materiales; conocidas éstas, podrán deducirse los complejos factores que orientarán en la fabricación de refractarios específicos para cada función en esta industria. Es muy posible que ciertas exigencias sean difíciles de lograr, pero ello representaría nuevos problemas que motivarían una vez más la necesidad de la investigación.

Dentro de la tecnología del cemento, en el campo de los refractarios existen las más variadas opiniones en cuanto a las propiedades o condiciones que deben reunir estos materiales para conseguir resultados afortunados, lo cual es explicable si consideramos la variedad de circurstancias, variables en cada caso, que concurren en el proceso de cocción.

En líneas generales, aun cuando en todo el horno-centrándonos ya en el rotatorio-existen problemas de refractario, donde las condiciones son más severas y los problemas más complejos es en la zona de sinterización. En esta zona, las materias primas en proceso de cocción permanecen de 15 a 20 minutos a $1.400-1.450^{\circ} \mathrm{C}$ y forman el clínker que desde ese momento empieza su período de enfriamiento.

La zona de sinterización se forra, normalmente, con refractarios extra-aluminosos o con refractarios básicos; la naturaleza del clínker de cemento ( $2 / 3$ de cal y $20-30 \%$ de fase líquida) obliga a este carácter básico del refractario por su menor reactividad. 
En la marcha normal del horno el material a cocer p-ovoca, normalmente, un pegamento al forro refractario for. mando una costra de clínker de varios centimetros de espesor y de cuya formación y permanencia depende, en gran parte, como veremos más adelante, la vida del revestimiento.

\section{Destrucción química del ladrillo refractario}

Como ya hemos dicho, la importancia técnico-económica del comportamiento de los reiractarios en la zona de cocción de los hornos rotatorios de cemento es tal que, en los últimos años, se han publicado gran número de trabajos de técnlcos e investigadores, tratando de destecar las propiedades más importantes del ladrillo. Todos ellos fijan una serie de ellas, generalmente las mismas, que en el estado actual de la técnica es poco menos que imposible reunirlas dentro de un mismo material.

Entre ellas presenta un interés especial, y a ello varros a dedicar nuestra atención, ei comportamiento fisicoquímico del crudo en reacción frente al revestimiento. Ccmo materiales de distinta naturaleza quimico-mineralógica, una vez enfrentados a la temperatura de trabajo del horno, constituirán un sistema en evolución. cuyo final será la destrucción del refractario con pérdida de sus propiedades esenciales.

I a corrosión química del ladrillo podemos decir que es la causa más generat, importante $\mathrm{y}$ continuada del des. gaste del revestimiento refractario. Su deserrollo dependerá de varios factores, entre otros los siguientes:

1. Naturaleza de los materiales (ladrillo y agente agresivo),-Como regla general, ambos serán lo más análogos posible considerados desde el ptunto de vista químico, aunque esta regla pueda sufrir excepciones al poseer el ladrillo otras propiedades de tipo físico que atenúen el fenomeno de corrosión quimica.

2." Temperatura.-Ya sabemos que la velocidad de reacción aumenta según una ley exponencial al crecer la temperatura, tal como enseñan las leyes de la Mecánica Quimica. Un aumento de temperatura, por otra parte, hace aparecer fases liquidas que favorecen considerablemente el ataque, al ser éste un medio más propicio a la reacción que el estado sólido e, incluso, formar parte en dicha reacción. Este es un peligro general en todos los laditillos, pero, sobre todo, en aquellos que por su composicion presenten eutécticos que proporcionan liquidos poco viscosos a temperaturas relativamente bajas.

3. Estructura fisica del ladrillo.-Sabemos que los poros y fisuras son vias naturales de penetración de los agen tes agresivos; la compacidad, por lo tanto, es un medio dei que disponemos para luchar contra la corrosión.

La fase vitrea es siempre más debll que la cristalina frente al calor; esto debe considerarse al preparar la pleza refrectaria.

4." Heterogeneidad físico-químiea del ladrillo.-Esta es siempre perjudicial; este factor tiene importancia al con. siderar el agtomerante del ladrillo y los materiales para juntas.

\section{Estudio de fases}

Si queremos dar una interpretación físico-quimica al fenómeno de la corrosión, es obligado recurrir a los diagra. mas de equilibrio a altas temperaturas, basados en Ias reglas de las fases.

Estos diagramas, como sabemos, reférense sólo a situaciones ideales-sustancias puras, atmósfera de reacción tnerte, equilibrio según las leyes de la Mecánica Química, etc. que, aunque distanciadas más o menos de la reali dad, permiten, en principio, hacerse una idea de cómo acontecerá un fenómeno aún cuando sólo sea en su aspecto cualitativo. Con esto disponemos de una base muy uitil para resolver, en primera aproximacion, muchos problemas de corrosión a altas temperaturas, que la experiencia conflrmará en último término.

La mayor parte de los procesos cerámicos y similares-el mismo cemento, por ejemplo-se encuentran en este caso; sus reacciones son incompletas y el resultado dependerá de las temperaturas alcanzadas y del tiempo de reac ción princtpalmente.

Por eso, al aplicar estos diagramas a cualquiera de estos procesos, debemos considerar detenidamente las posibles diferencies entre las condiciones ideales y las reales. Por ejemplo, si nosotros consideramos un cuerpo ciraiquiera formado, para simplificar, a partir de materias primas que en su constitución presentan solo tres oxidos, al calentar suficientemente la mezela e, incluso, llegando a la fusión parcial, podremos dar la composición resultante haciendo uso de los diagramas. Es casi seguro que las fases observadas no corresponden cuantitativamente ri, a veces, ctalitativamente, con los constituyentes encontrados microscópicamente en un producto comercial de la misma compostelón.

Vemos que, por desgracia, nos faltan resortes en la interpretación y éstos son los factores dinámicos de las multiples reacciones que determinan el producto final. Los diagramas de equilfbrio no hablan del camino recorrido para conseguirlo $y$, por lo tanto, no dan ninguna información acerca de velocidades de reacción tan dificiles de pre- 
ver en conglomerados heterogéneos $y$ en estados de egregación que oscilan del solldo cristalino al liquido flúido e, incluso, hasta el gaseoso. Si a ello sumamos otros factores, tales como viscosidad de los componentes liquidos, velocidad de disolución de sólidos y difusión de los materiales en su conjunto, comprenderemos que el final es difí cll de predecir, el equilibrio diffcll de lograr e, incluso, la homogeneidad del material dudosa de ser perfecta.

Por esto, insistimos que al hacer aplicación de los diagramas deben cuidarse mucho la relación entre las condiciones actuales $\mathrm{y}$ las ideales: temperaturas alcanzadas, thempo de tratamiento, naturaleza de las impurezas, etc.

El sistema base de todos los estudios sobre refractarios y sus relaciones con las materias primas del slínker de cemento, en fase de reacción variable, es el de cinco componentes:

\section{S.A-F-C-M,}

el que, a su vez, Iógicamente lleva consigo el conocimiento de otros más sencillos.

El diagrama S-C-A.F, de aplicación a refractarios aluminosos, está muy estudiado por los investigadores de cemento. Por otro lado, Rait y sus colaboradores han hecho estudios sobre aquellos diagramas que comprenden los refractarios básicos de magnesita, cromlta, dolomita y forstertita.

Un punto importante en la observación de diagramas, es la temperatura a la que se forma el primer lfquido fundido-que en la practica suele ser un poco inferior al no considerar nuevos componentes que son las impurezas $y$ que casl siempre rebajan el punto de fusión-y las cantidades desarrolladas en función de la temperatura; no deben de olvidarse las características de estos liquidos en lo que se reflere a viscosidad, composición, etc., y ast, por ejemplo, los que proceden de ladrilios bésicos son muy flíidos en relación con los que resultan en los materiales siliceos.

Esta fase líquida permite una mayor aproximación a las condlciones de equilibrio. Los refractarios se suelen fabricar a temperaturas por debajo de la completa fusión y son solo stnterizados, es decir, calentados a temperaturas en las que sólo una pequeña porción de la masa se encuentra en estado líquido. Sin embargo, hay casos, como los ladrilios básicos, en que muchos autores suponen que su constitución puede deducirse de los datos de equilibrio.

En el ladrillo acabado, la fase vitrea-función del porcentaje de lfquido en la sinterización-resiste menos la corrosión quimica que la fase cristalina $\mathrm{y}$, en general, el mecanismo de ciertas propiedades físicas y de los fenomenos mecánico-destructivos se encuentran ligados con el estado de los componentes mineralógicos.

Sobre el clinker, como ya hemos dicho, se han hecho ya estudios muy completos sobre las fases presentes en las condiciones industriales de desequilibrio parcial en el sistema S-C-A.F.

Las relaciones ladrillo-clínker, interpretadas según los diagramas de fases, han sido estudiadas, teortea y prácticamente, por varios autores.

Lerch, por ejernplo, considerando tos diagramas ternarios S-C-A y S-C-M, observo la superioridad quimica del forto de magnesita frente a los materiales sflicoaluminosos; esta superioridad se reflere, fundamentalmente, a las temperaturas en que aparecen fases liquidas, atravesando zonas ternarias con eutécticos próximos de puntos de fusión bajos, en el caso de los materiales ajuminosos. La escala en que se evicencia esto en ta realidad, lo ponen de maniflesto los estudios microscópicos y roentgenográflcos determinando los minerales formados en la zona de contacto ladrillo-clinker, como veremos mas adelante.

Observamos en el diagrama de la figura 1 que los ladrillos aluminosos se encuentran en tn desequilibrio peligroso rente al clínker y su corrosión seria brusca y continuada si no fuese por otras circunstanclas que lo impiden-formación de costra, por ejemplo-

Los ladrillos de magnesia, sin embargo (fig. 2), parecen muy estables teóricamente frente a los efectos de la fase vitrea del clínker, pero en la práctica no son tan inactivos como parece a primera vista: esta fase vitrea penetra en el ladrillo a través de los poros, y aunque no puede reaccionar químicamente en gran escala con la periclasa (ni quizás con el ferrtto magnésfoo en ella disuelto), si puede hacerlo con la sustancia de fondo o intersticial, donde se encuentran las impurezas que no hemos considerado en el diagrama anterior.

Como ven, es dificil saber, en cada caso, hasta qué limites pueden aplicarse los diagramas de fases.

En todo caso, el tiempo necesario para llegar al equilibrio dependerã de varios factores; entre otros:

a) Superficie de contacto entre los componentes del sistema.

b) Medida en que se separan los productos de reacción una vez formados, dependiendo de ia velocidad de difusión que, lógicamente, será mayor si el producto es flúido. 

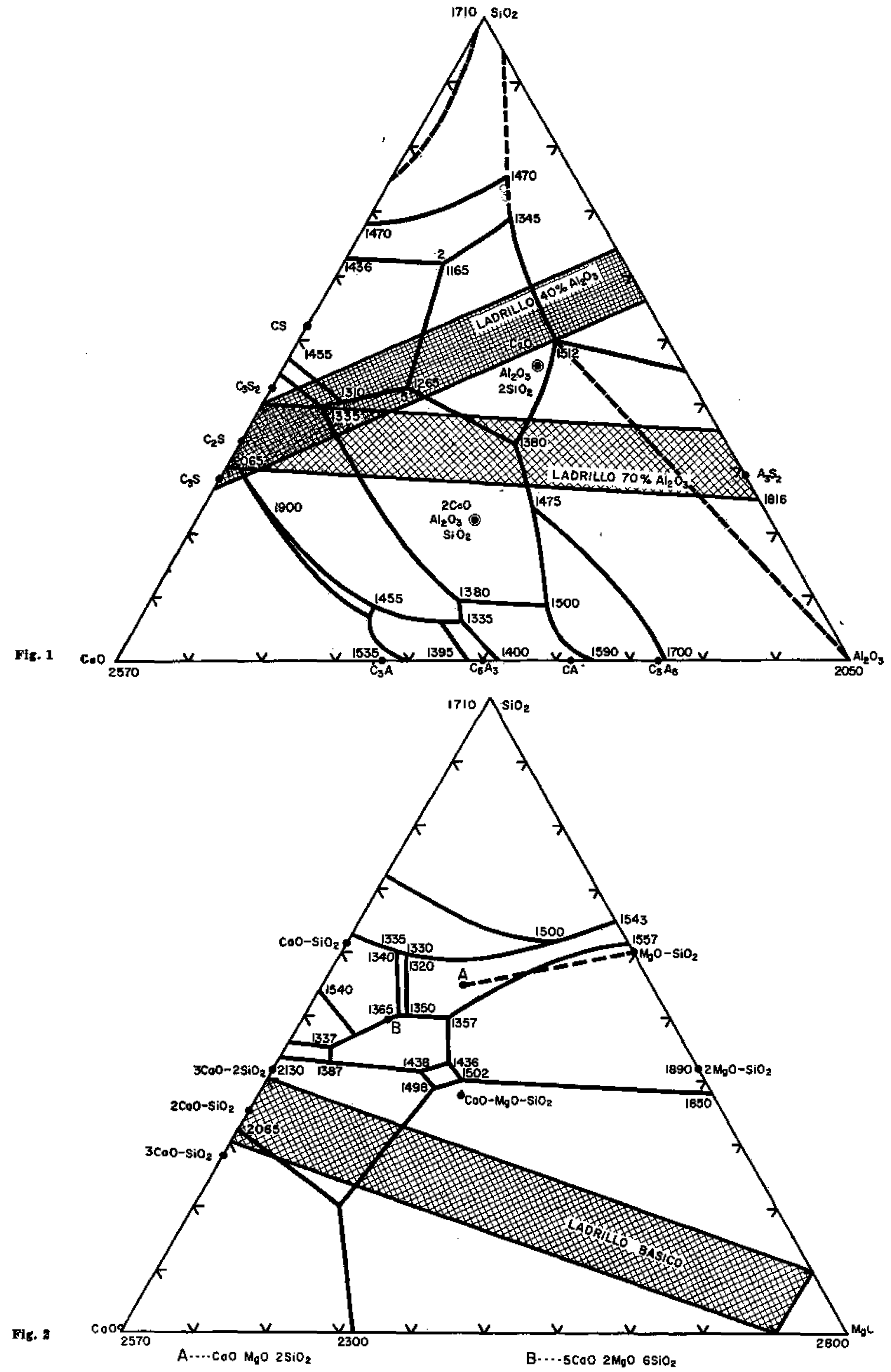


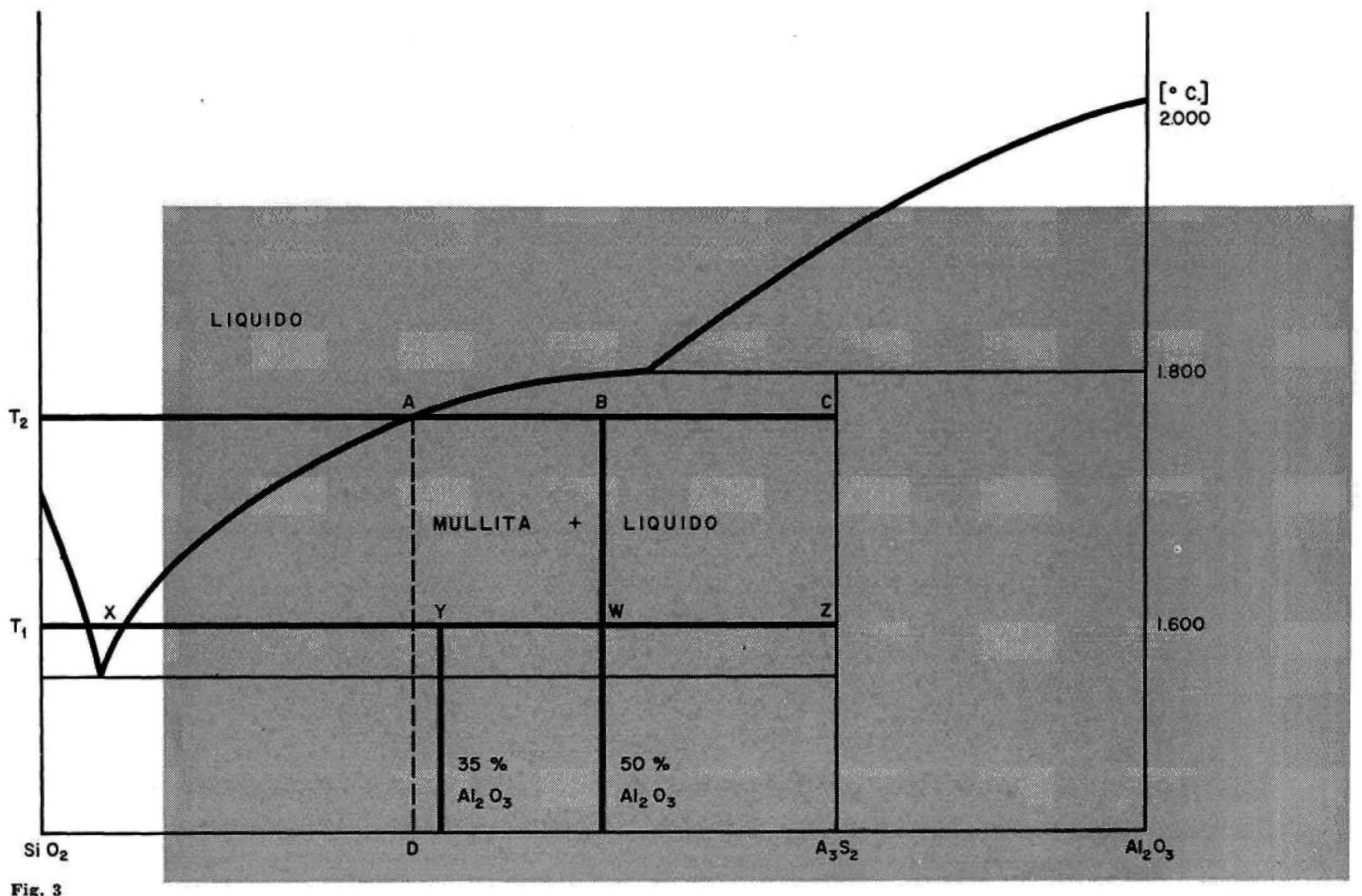

Fig. 3

c) Energía de activación de los productos reaccionantes.

d) Energia de combinación (con sus posibles influencias en la temperatura).

e) Posible disociación del producto formado y extensión en que ocurre a la temperatura de trabajo.

Finalmente, siempre nos queda un consuelo: la formación de costra, de la que hablaremos después, y el gradual enfriamiento de la zona de contacto que frena esta velocidad de reacción, la cual, posiblemente, continuará, pero mucho más lentamente.

\section{Mecanismo del ataque químico}

Vista la posibilidad de la corrosión, vamos a extendernos un poco en el posible mecanismo del ataque.

La fase líquida del clínker se absorbe en los poros del refractario $\mathrm{y}$, entonces, puede reaccionar con las fases constituyentes del mismo, siendo lógicamente la más susceptible la fase vítrea o líquida. Al ponerse en contacto los dos líquidos se mezclan por difusión, la velocidad de la cual crecerá exponencialmente con la temperatura y disminuirá al aumentar la viscosidad.

La fase liquida del clínker es, normalmente, más flúida que la fase vitrea del refractario, la cual, por lógica, debe ser viscosa para que el ladrillo tenga la resistencia mecánica requerida a altas temperaturas. Si esta fase liquida se diluye con la del clínker, la viscosidad total disminuirá y, eventualmente, el ladrillo caerá, o, si se trata de un horno rotatorio, la porción afectada será arrastrada. La fase cristalina se atacará más lentamente, en parte porque está rodeada de fase vítrea y, en parte, por ser más refractaria.

Supuesto este, el mecanismo del ataque, a igualdad de otras condiciones, las posibilidades de corrosión crecerán con la porosidad, pero ésta tiene limitada su reducción sí tenemos en cuenta la necesidad de la resistencia al choque térmico con la que debe mantener un compromiso dicha porosidad. La selección de materias primas y las técnicas de fabricación de ladrillos tienen la palabra para aquilatar al máximo en este compromiso. 


\section{Refractarios sílieo-aluminosos}

Supongamos dos materiales con contenidos de alümina del 35 y del $50 \%$, respectivamente (fig. 3). Si los cocemos para conseguir un estado similar de resistencia mecánica en frio y porosidad, a igualdad de otras circunstancias, habremos conseguido la formación de cantidades similares de fase liquida. Para lograr esto en el material más aluminoso, necesitaremos una temperatura $T$, superior a la $T$, para el otro material. En el equilibrio, las cantidades de líquido vendrán dadas por las relaciones $\mathrm{BC}: \mathrm{AC}$ e $\mathrm{YZ}: \mathrm{XZ}$.

A una temperatura de trabajo del ladrilio inferior a $\mathbf{T}_{1} \circ \mathbf{T}_{2,}$ el aglomerante vítreo del ladrillo más aluminoso estara más superenfriado que el del menos aluminoso, y su punto de reblandecimiento será más elevado $\mathrm{y}$, en consecuencia, mís resistente a la carga a las altas temperaturas.

El caso seria el mismo para dos materiales de igual contenido en alúmina, pero con diferente tipo o cantidad de impurezas (álcalis, cal, oxidos de hierro, etc.): la fase lfquida del ladrillo preparado con materlales menos puros tendra una temperatura de reblandecimiento menor.

Ahora bien, como en el enfriamiento la fase vitrea puede desvitrificarse, en parte, un ladrillo frío puede dar una idea muy inexacta de sus condiciones a las temperaturas de trabajo; al elevar su temperatura de nuevo, las fases que han cristalizado se redisolverán.

A una temperatura de trabajo $T_{1}$ el ladrillo de $35 \%$ de alúmina volverá a adquirì su relación liquido/solido original de $\mathrm{YZ} / \mathrm{XY}$, pero en el de $50 \%$ la relación será solo de $\mathrm{WZ} / \mathrm{XW}$. Si existen impurezas, a una temperatura dada, la proporción de líquido será mayor en el ladrillo menos puro.

En resumen, los refractarios con mayor contenido de alúmina o con menor contenido de impurezas tendrán, normaImente, un aglomerante vitreo en menor proporción o con un punto de reblandecimiento más elevado, al someterlos a una temperatura de trabajo dada.

Hasta aquí hemos hecho un esquema simplificado aplicado sólo al ladrillo. Ahora bien, si tomamos en lugar del diagrama binario S-A, el ternario S-A-C en el que con cierta aproximación pueden representarse simuitáneamente el clínker de cemento y el refractario silicoaluminoso, comprenderemos todavía mejor la importancla de la fase liquida.

En la figura 4, la zona $A$ representa la composición del cemento, y la $B$, un ladrillo de $60 \%$ de alumina con una pequeña cantidad de cal como impureza. Como en planta estos diagramas representan composiciones, es una técnica muy usada, como ya hemos dicho, trazar una línea que una las dos composiciones y anotar las isotermas de solidificación por las que atravlesa.

Para interpretar los hechos es mejor considerar las composiciones de las fases liquidas que entran en juego, supuesto que es muy instructivo considerar que el proceso de reacción ocurre en tres fases:

1." Penetración de la fase liquida del clínker en los poros del ladrillo.

2." Mezcla por affusión de este líquido con la fase líquida del ladrillo.

3." Reacción o solución por el nuevo líquido con las fases sólidas del ladrillo.

Estos procesos ccurrirán simultáneamente en alguna extensión, pero hay evidencia práctica, al menos con ladrillos básicos, de que está justificado este método deductivo.

La composición del liquido del clínker yace en el campo de cristalización primario del sillicato tricálcico sobre la isoterma de $1.500^{\circ} \mathrm{C}$, puesto que de momento ignoramos las impurezas que rebajarfan la temperatura de clinkerización.

La composición de la fase líquida de ladrillo puede considerarse en $\mathrm{D}$, resultando un $20 \%$ del ladritlo como fase liquida. Este porcentaje no se afecta muy directamente por el contenido de alúmina, pero sí por la temperatura de cocción, fijada Indirectamente por el contenido de alúmina y las impurezas.

Si observamos el diagrama, $\mathrm{AB}$ en su porción estrecha a la isoterma $1.400^{\circ} \mathrm{C}$. pero $\mathrm{CD}$ (recta de unín de las dos fases liqułdas) atraviesa esta área y pasa muy próxima a la isoterma $1.300^{\circ} \mathrm{C}$. Cuando el ladrillo absorba el liquido del clínker, la nueva fase líquida tendrá una composición que se encontrará en la zona rayada y señalada con la letra $\mathrm{E}$.

E1 ladrillo contiene alrededor del $20 \%$ de liquido. Si su porosidad es también del $20 \%$ y los poros se llenan con la fase lfquida del clínker, $P$ representara la mezcla líquida resultante. La composición final del fadrillo, más la fase liquida del clínker seŕ́ $Q$, intersección de $\mathrm{BC}$ y $P-\mathrm{A}_{2} \mathrm{~S}_{3}$; al mezclarse los dos líquídos reaccionarán con la mulita, a la cual suponemos la fase sólide del ladriilo. 


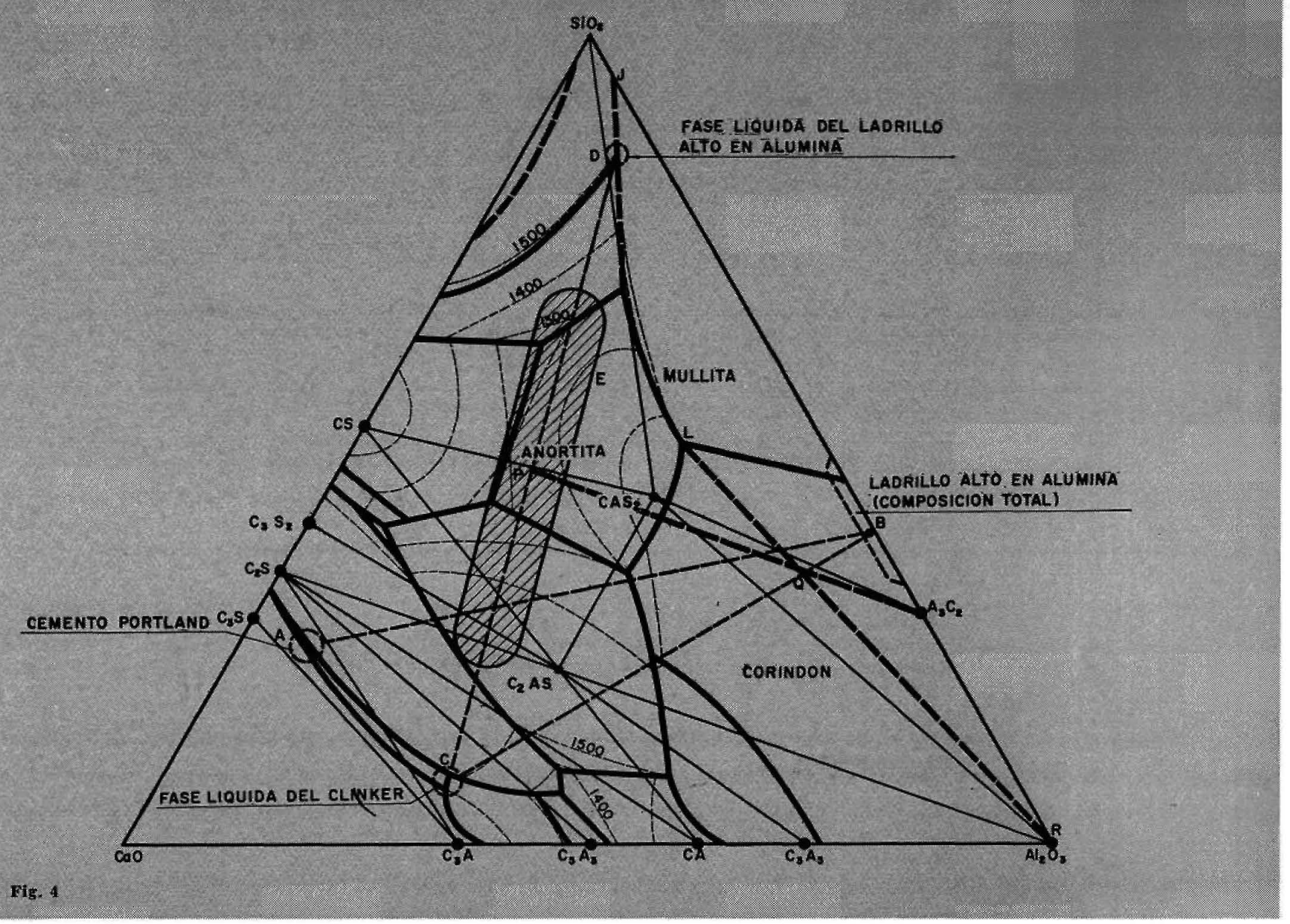

La reacción de la mullita con el líquido $\mathrm{P}$ resultará en una mezcla de corindón sólido más un líquido de com. posición L, en las proporciones de, aproximadamente, $70 \%$ de líquido y $30 \%$ de sólido, al sobrepasar $1051.500^{\circ} \mathbf{C}$ (exactamente $1.512^{\circ} \mathrm{C}$, punto de fusión del eutéctico $\mathrm{L}$ ).

En la práctica, por la presencia de impurezas, se reducen las temperaturas de los puntos invariantes del sistema ternario.

De este modo, la "solubilidad" final del ladrillo es muy grande y la corrosión acentuada.

\section{Refractarios básicos}

Para estos ladrillos es más difícil hacer uso de los diegramas de fases. La característica más importante del sistema S-C-A-M es que gran parte del tetraedro está ocupado por el volumen de la fase primaria periclasa, y los correspondientes planos isotermos marchan bastante pararelos hacia el punto que representa la fase líquida del clínker. Por otro lado, se ha encontrado experimentalmente que la solubilidad de la magnesia en la fase líquida del clínker es sólo del 5-6\% a $1.400-1.450^{\circ} \mathrm{C}$.

Este efecto se demuestra ya incluso en el diagrama triangular S-C-M, donde el área de la periclase es grande y próxima al área de las composiciones del clínker.

La fase líquida en los ladrillos de magnesia se encuentra aproximadamente en la línea que une el silicato bicálcico con la forsterita, encontrándose el punto de solidificación más bajo en las proximidades de la monticelita (véase fig. 2).

En los ladrillos de cromo-magnesita la situación es más compleja, puesto que su representación adecuada reque ríría un diagrama de cinco o seis componentes. 


\section{Estudio experimental del fenómeno de la corrosión química}

Varios autores se han ocupado de estudiar los fenomenos que tienen Iugar en la superficie del revestimiento refractario, después de usado en un horno rotatorio de cemento.

Las técnicas empleadas generaimente han sido la observación macro- y microscópica y el estudio roentgenografico. En ladrillos de $40-45 \%$ de alúmina, Ia zona de soldadura ladrillo-costra dio como fases resultantes el silicato bicálcico, la ghelenita y la anortita. En los de- tipo $75 \%$ de alümina, resultaban abundantes cristales de ghelenita y masa vítrea.

Como se puede observar, esto está de acuerdo con las interpretactones dadas con los diagramas de fases.

Mencionemos ahora dos casos, corrientes en la realidad de la práctica, en los que parece encontrarse resultados paradójicos si interpretamos los hechos con la base teórica desarrollada hasta el momento.

El primero hace referencla a dos refractarios aluminosos empleados en análogas circunstancias, el más bajo en alümina de los cuales resistió más tiempo en uso que el alto que presentaba textura homogénea.

La explicación que da el autor de esa publicación es que en el primer caso el clínker provoca rápidamente un producto de reacción que penetra entre los gránulos de chamota en forma sinuosa, favoreciendo la adherencia al refractario no sólo del producto de reacción, sino también de la costra de clinker, rebajando la temperatura en la zona de contacto e impldiendo que la corrosión progrese velozmente. No ocurre lo mismo con el refractario de textura homogénea, en los que entre la costra y el refractario existe un estrato mucho más regular y uniforme que el anterior.

El autor de estas experienclas (doctor Goria) atribuye mayor adhereneia al hecho de que las líneas de isocomposición son sinuosas y no coinciden con las lineas isotermas y que, naturalmente, cuanto más diferentes sean estas líneas, la adherencia será más fuerte y el proceso de corrosión más lento.

Este autor, en sus estudios experimentales de fases ha observado una absorción preferencial de la magnesia respecto a la cal đel clínker y ha individualizado la formación de una solución sólida ghelenita-akermanita y notable masa vitrea de idéntica composición.

El segundo caso, mencionzdo por otro autor italiano (doctor A. Coceo), relata el comportamiento de dos refractarios de composición química mily analoga, empleados en dos hornos contiguos en marcha normal y cociendo el mismo clínker. Su composiclón qúmica era la siguiente:

$\begin{array}{ccc}\text { Composieión } & \begin{array}{c}\text { Refractario A } \\ \%\end{array} & \begin{array}{c}\text { Refractarko } \\ \%\end{array} \\ \mathrm{SiO}_{2} & 29,3 & 28,3 \\ \mathrm{Al}_{2} \mathrm{O}_{3} & 67,9 & 66,9 \\ \mathrm{TiO}_{2} & 0,9 & 1,4 \\ \mathrm{Fe}_{2} \mathrm{O}_{3}+\mathrm{FeO} & 1,75 & 3,2\end{array}$

Ei refractario A, en su estructura, presenta alrededor de un $50 \%$ de un material, procedente probablemente de caolinita enriquecida en aluminio y una masa de fondo procedente de un material bauxitico muy rico en alúmina. En el empleo da un buen resultado y presenta una zona alterada de unos dos centimetros de espesor diversamente coloreada, cuyo estudio en el laboratorio dio los siguientes resultados:

Refractario no alteracio.
Estructura norma:

Estructura más homogénea.

Gránulos de chamota (Corindón).

Vidrio.

Corinoon-Hexaaluminato cálcico.

Masa amorfa y probable ghelenita.

Anortita.

Ghelenita.

Ghelenita-C, $\mathrm{C}-$, fases no individualizadas. 


\section{Zona marrón:}

Silicato bicalcico.

Fase no individualizada.

Ghelenita.

Zong alterada

\section{Zona rosa:}

Silicato bicálcico (abundante).

Material poco reflector, en general gelatinoso.

Zona verde:

Silicato bici]lcico.

Material poco reflector gelatinoso.

Costra de clínker.

En la tabla siguiente se encuentran los análisis químicos de los tres estratos coloreados de la zona alterada:

COMPOSICION

$$
\mathrm{SiO}_{2}
$$

$\mathrm{Al}_{2} \mathrm{O}, \mathrm{com}$.

$\mathrm{Fe}_{2} \mathrm{O}_{\mathrm{a}}$

$\mathrm{FeO}$

$\mathrm{CaO}$

MgO

Alcalis
MARRON

26,5

27

0,3

0,7

37,5

2,5

5
\% EN PESO

ROSA

VERDE

30,3

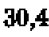

11

0,35

5

$0,25-0,6$

51,8

57

1,6

1,1

3

Es interesante observar microscópicamente la zona de paso entre el refractario alterado y el no alterado; el complejo presenta alrededor de siete componentes, de los cuales el más abundante es la ghelenita. La mayor parte de ellos no respetan las condiciones de equilibrio e, il cluso, no pueden coexistír en equilibrio entre sf. Si recuece el material sels horas a $1.400^{\circ} \mathrm{C}$, el conjunto se reduce a dos fases; ghelenita (abundante) y masa vitrea.

El refractario $B$ ha dado un resultado desastroso en su emplei. Al cabo de veinticuatro horas ha quedado casi destruido por el clínker, y hacia la zona caliente presenta una zona oscura de espesor no superior a 162 mm.

En su estructura original se distinguen tres zonas: una primera, de gránulos de bauxita calcinada; otra, de grá. nulos de materlal sillcoaluminoso, y la tercera, de una rrasa aglomerante de naturaleza sillicoaluminosa también.

La estructura del refractario alterado se encuentra en la siguiente tabla;

Refractario no alterado.

\section{Zona A.}

Zonas B y C.

En el límite con el refractario.

Zona alterada

Hacia el exterior.
Corindón.

MulHta y vidrio.

Corindón-Mullita-Vidrio (más abundante que en el refractario no alterado).

Corindón-Vidrio y pequeña cantidad de anortita.

Ghelenite-Vidrio.

Corindón.

Es interesante observar que en la zona de paso la fase vitrea aumenta a medida que nos acercamos al borde 


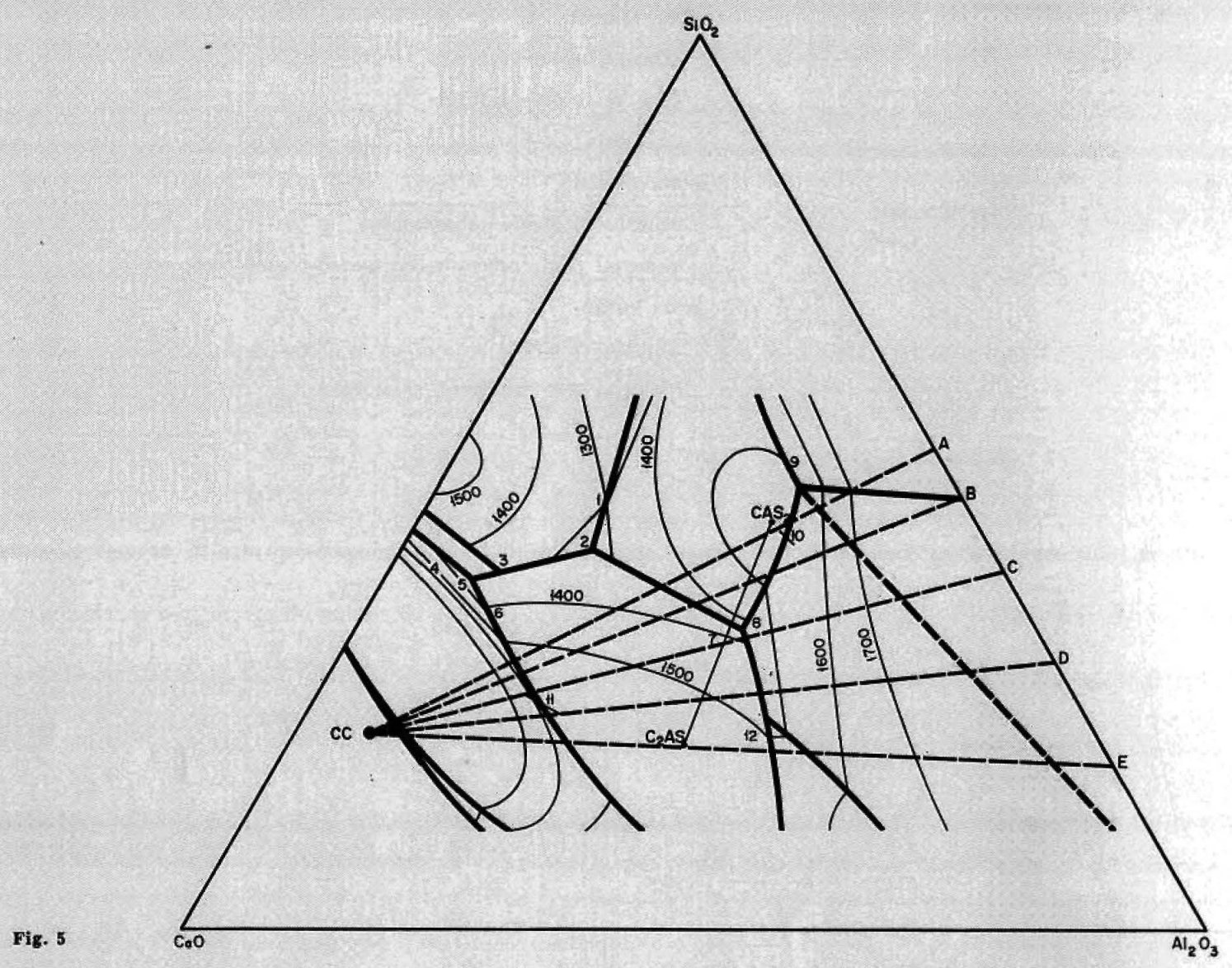

Expliquemos un poco este segundo caso sobre un diagrama ternario, S-C-A (fig. 5), donde representamos, de una parte, el cemento, y de la otra, la masa total del refractario (D) y la masa total del refractario excluído el corindón (B). De la observación experimental (véase cuadro anterior) resulta claro que no todos los componentes del ladrillo reaccionan simultánea y totalmente con el clínler.

Es evidente, que la composición del refractario que ha reaccionado para formar la zona del vidrio en el límite con la costra está comprendida entre las relaciones S/A de la masa total del refractario y la de esta masa excluída del corindón. Esto se confirma con dos hechos experimertales: de una parte, la falta de mullita nos dice que la parte del refractario compuesta de mullita y vidrio ha reaccionado, y de la otra, la presencia de corindón indica que la masa reaccionante no ha alcanzado todavia la relación S/A de la masa total del refractario. La misma consideración puede hacerse con la segunda capa, donde, además de ghelenita y vidrio, sigue existiendo corindón.

Este es, ciertamente, el último en reaccionar, y puede asegurarse que no llega a reaccionar del todo, como lo demuestran los gránulos de corindón encontrados en la costra, parcialmente arrastrados por la acción corrosiva del clínker sobre el refractario.

Veamos ahora las conclusiones a que se llegan para explicar el comportamiento tan distinto de estos dos refractarios.

Dadas las circunstancias tan iguales en la marcha de los dos hornos, debemos suponer que la causa está en el propio refractario. Una confirmación de esto son las pruebas realizadas en el laboratorio, que demuestran que el ladrillo B resiste más la acción corrosiva del cemento.

Para centrar el problema, comparemos la estructura de los dos refractarios. El primero está constitúdo por chamota de material arcilloso y bauxita calcinada como material aglomerante; el segundo, bauxita calcinada como material granular y material arcilloso como aglutinante. En otras palabras, tenemos dos refractarios en los que se han invertido el papel de los materiales empleados. A esto atribuye el autor del trabajo el mal comportamiento del refractario $B$. 
En efecto, para el primer refractario podemos decir que el corindón, por su estado de división elevado, ha reacclonado rápjda y totelmente, junto con el resto de ja mesa refractaria, al ponerse en contacto con el clínker. Esto ha conducido a una varlación de la composición química de la superficie reaccionante a lo largo del segmento que une la relación $\mathrm{S} / \mathrm{A}$ de la masa total del refractarto con la composición del clinker.

En el segundo, las cosas ccurren de un modo muy distinto. Aqú, el corindón, al encontrarse formado por grá. nulos compactos, ha permanecido en gran parte como tal. De esto resulta que la composición de la masa reaccio nante no se ha movido a lo largo del segmento que une la relación $S / A$ del refractario con la composición del clínker, sino que se ha situado a lo largo de un seginento que une la composición del clínker con la relación $S / A$ comprendida entre la de la masa total del refractario y la de esta masa excluido el corindón.

La consecuencia de todo esto puede deducirse de las observaciones experimentales y del examen del diagrama ternario S-C-A. En efecto, en el caso del primer ledrillo el segmento en que se sitúa la zona alterada pasa próximo al punto de composición de la ghelenita. Por lo tanto, se forma un porcentaje elevado de este compuesto, acompañado de una cantidad no muy elevada de fase furdida. Fin el segundo caso, el segmento que une las composiciones medias de las masas reaccionantes pasa próximo al eutéctico ghelenlta-anortlta (punto C).

Es evidente que en estas condiciones el porcentaje de fase fundida en las condiciones de trabajo es, I 6 gicamente, elevada, como confirman las observaciones experimentales. Este vidrio diffulta la adherencia y, en consecuencia, el ladrillo permanece desnudo y se corroe rápidamente.

\section{Formación de costra protectora del ladrillo refractario}

Un aspecto importante en el buen funcionamiento de los hornos $y$ en la estabilidad del revestimiento es la tendencia del clinker a adherirse al refractario, singularmente en la zona de sinterización.

Es casi general la opinfón de que la formaolón de esta adherencia en la puesta en marcha de un horno es la base para la durabilidad \& estabilidad de cualquier revestimiento en tanto no se esté en condiciones de fabricar refractarios más perfectos y resistentes a las altas temperaturas $y$, sobre todo, a los procesos que tienen lugar durante el funcionamiento. Es decir, al forro refractarlo se le reserva la misión de soporte y la costra hace las veces de un verdadero revestimiento.

Sobre el mecantsmo de formacion de esta costra hay diversas opintones, pero que pueden resumirse en dos procesos fundamentales: adherencia físlca y reacción química.

Unos autores (Heuenschild, por ejemplo) ven la causa de formación y renovación de la adherencia en un ciclo de solidificación y fusión del clínker con la oscilación periódica de la temperatura. Esta teoria se ve apoyada por el siguiente hecho: los ladrillos de magnesita enşayados con un clínker de laboratorio no se atacan, quedando aquél como una masa sinterizada en el fondo de la oquedad previamente preparada; sin embargo, en la realidad casi siempre se produce adherencia, lo que demuestra que la carga de cliniker por sí sola desarrolla un poder aglomerante tal que se adhiere al ladrillo, aunque sólo sea por un fenómeno físico.

Spangenberg atribuye el fenómeno a una reacción quimica de la fase vítrea del clinker con el refractario. En Ios forros de chamota o extraaluminosos esto se da sin más, pero en los de magnesia es posible que sólo ocurra esto en ciertas composiciones favorables de crudo.

Segin Mussgnug, el factor decisivo para crear un revestimiento protector es la propia composición química del crudo, atribuyendo especial importancia a las relaciones S/A y A/F, deducides del análisis del clinker. Al $6 \times i d o$ manganoso se le considera englobado con los oxidos de tierro y no considera más componentes, aunque algunos, como alcalis y sulfatos, puedan perjudicar al forro si no hay suficiente costra formadk.

Considera que los valores optimos de estos indices son los siguientes:

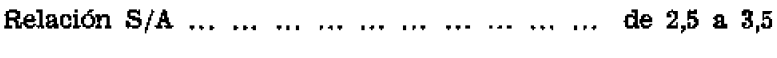

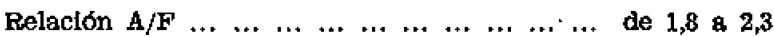

La costra se altera en el sentido:

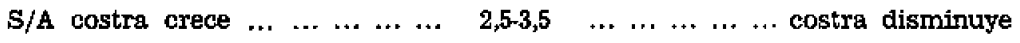

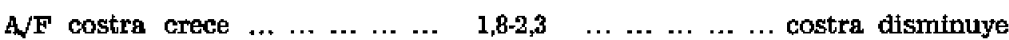

Si se mantfene el módulo stlícico, la costra crece al disminulr el módulo de alúmina, el cual tiene siempre une influencia más marcada que aquél. 


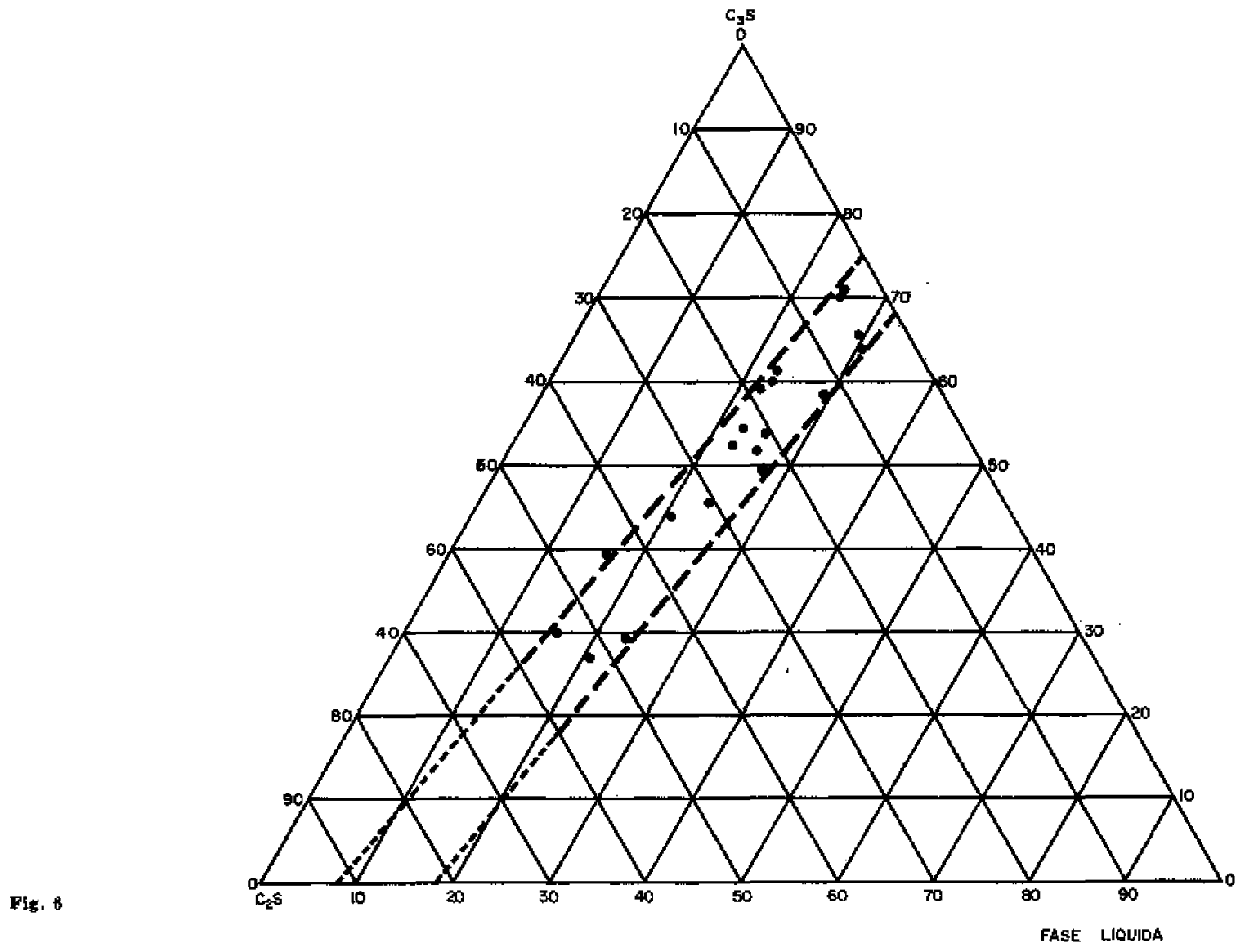

Más tarde, Ia tesis de Mussgnug he sufrido ampliaciones con los estudios de Konopicky, quien relaciona el esta. do físico del clínker en fase de sinterización con la composición quimicomineralógica del mismo. De suts conocimientos en la investigación de sistemas distintos a los del cemento en movimiento rotatorio, y por analogia, deduce que sólo la «viscosidad del crudo en fase de sinterización es causa de la formación de adherencias para una determinada zona de porcentajes de fase liquida, excluyeado las teorias de solidificación y adhesión química sobre el forro.

Con esta base y sus investigaciones en clinkeres comerciales deduce, graficamente (fig. 6), los limites permisibles de fase líquida en cada caso, la cual es máxima para clinkeres ricos en silicato tricalcico (alrededor del $30 \%$ ), disminuyendo al crecer el porcentaje de silicato bicálcico. Es decir, Ios clínkeres ricos en bicalcico requieren menos fase liquida, obrando equel como parte integrante de la misma, lo que se atribuye a su elevada tendencia a la recristatización por debajo del punto de fusión. Al crecer el contenido en oxido de hierro, disminuye la porción necesaria de fase líquida, explicable por provocar un descenso en la viscosidad de ésta.

Con todo, podemos decir que la formación de adherenoia y sus características son función de la composición quimico-mineralogica, tanto del crudo como del refractario $\mathrm{y}$ de su comportamiento con la temperatura, responsables ambos de la formación de fase lfquida y de las ar racterísticas de la misma. Fn otras palabras, a cada com posición de crudos-a igualdad de refrectario-corresponde una temperatura determinada para que la íneriastación se forme y otra para que desaparezca.

En ocasiones, estos areblandecimientos" optimos coinciden en una zona tan estrecha de temperatura que en la práctica puede no ser alcanzada o rebasarse, alternativamente, dentro de una revolución del horno. Fn estos casos, por descracia, los límites de composictón fifados por Mussanug y otros autores no stempre pueden manejarse fácil mente en la industria del cemento Portland, limitados a materlas primas que se mueven entre indices muy estrechos y disponiendo sólo de variaciones cómodas en el limite de saturación en cal.

El refractarlo influye en el sentido de modficar los indices de composición óptimos y les temperaturas adecuadas para una buena adherencla, presentando interes, en este aspecto, por el modo en que reblandece con la tempe ratura y la presión. Es más, es posible que haya una relación tan estrecha entre ios puntos típicos del ensayo de 
reblandecimiento de refractarios $\left(t, y t_{b}\right.$ ) y la temperatura óptima de sinterización del crudo de cemento, que resultaria superfiuo y hasta deswentajoso emplear ladrillos de refractariectad elevada; hablamos, claro estä, en el supuesto de buscar la formación de una adherencia estable.

En cada caso, se considerará como buen punto de referencia la temperatura de fusión más baja para las mezclas refractario-clinker molidos. Tschirky considera esta de $1,250^{\circ} \mathrm{C}$ para los ladrillos de chamota $(40 \%$ de alúmlna) y $1.400^{\circ} \mathrm{C}$ para los extraaluminosos (70\% de alúmina), ambas temperaturas inferiores a las de cocción de los clínkeres de hoy dia. Para los Iadrillos de magnesia resuitaban temperaturas superiores a Ios $1,800^{\circ} \mathbf{C}$, limite observable en el aparato de ensayo.

Según esto, el hecho de que haya fábricas con resultados satisfactorios empleando ladrillos de chamota se debe a que las condiciones de trabajo son tales que se forma un revestimiento de suflciente espesor $y$ bien adherido, rebajando tanto la temperatura a su través, que la temperatura en el contacto ladrillo-costra es inferíor a la temperatura ce fusión más baja de las mezclas pulverizadas ladrilla-clínker.

\section{Factores que modifican la adherencia}

En primer término, la conducción del horno y el proceso de combustión son del máximo interés en la formación, conservación y destrucción de la costra. En este sentido, la experiencia confirma que la conducción del fuego con llams larga es más sencilla que con llama corta y más asequible para formar un buen revestimiento; esto se debe a que la oscilación de temperatura en un gito del borao dentro de una sección es más pequeña (fig. 7).

Lógicamente, para cada caso habra un espesor optimo y un espesor minimo por debajo del cual siempre hay pérdida de revestimiento. Este fenómeno destructivo, gradual $y$ lento, va unido a la dispersión térmica a través de la chapa del horno, segundo factor de gran importancia.

La transmisión de calor al exterior es pequeña para espesores grandes de refractario, llegando a impedirse la formación de costra por exceso de temperatura en su cara interna. El forro desnudo, expuesto a todas las acciones -llama, crudo, gases, etc.-, fundiré y desgastará rápidamente hasta reducirse a un espesor tal que por radiación exterior enfría en su cabeza y se produzca la soldadura y formación posterior, lo que sólo es posible con un gradiente dado de temperatura a través del revestimlento. La experiencia señala 15 cm como el espesor más ventajoso en eil caso de ladrillos de chamota o aluminosos.

Resumiendo, podemos decir que la estabilidad de la costra y su relación con el refractario depende de dos tipos fundamentales de causas:

Unas intrínseeas, independientes de la marcha del horno, que son: los refractarios (calidad, espesor, etc.), los crudos (facilidad de sinterización, composición, etc.) y los combustibles (calfaad de las cenizas, tipo de Ilama, etc.).

Otras accidentales, como son las paradas del horno, las irregularidades de la alimentación del crudo (composición físico-química y cantidades), los defectos en la tnstalación del revestimiento y los golpes de fuego y enfriamientos durante la marcha.

\section{Como oprecior la corrosión química de un ladrillo refractario en el laboratorio}

Como acabamos de ver, el ataque de un material refractario por un agente corrosivo, como es el clínker de cemento Portland en grado de sinterización, es el resultado de la superposición de una serie de fenómenos físicos, químicos y mecánicos, dependientes, a su vez, de otras condiciones exteriores, tales como temperatura, tiempo, atmósfera del horno, etc.

Al ser numerosos los factores que intervienen en el desgaste, al buscar un ensayo de tipo tecnológico trataremos de acercarnos a las condiciones prácticas manefando como variables, o dando más importancia, al grupo de factores que se consideran más influyentes en la marcha del fenómeno corrosivo; es la diferencia con los ensayos de investigeción, en los que se tratan de separar estos factores, hacerles variar y sacar conclusiones sobre la teoria del fenómeno.

De un modo general, los factores más importantes en la destrucción química del ladrillo son:

a) Las características físicas $\mathrm{y}$ químicas del refractarlo $\mathrm{y}$ del clínker.

b) La velocidad de reacción entre el refractario y los componentes del clínker.

c) La velocidad en la aportación de clínker a la zcna de reacción.

d) La velocidad en la eliminación de los productos de reacción. 
TEMPERATURAS DE LA PARED INTERNA EN HORNOS ROTATORIOS DE CEMENTO

CON ZONAS DE SINTERIZACION LARGAS Y CORTAS(SEGUN HAUENSCHILD)

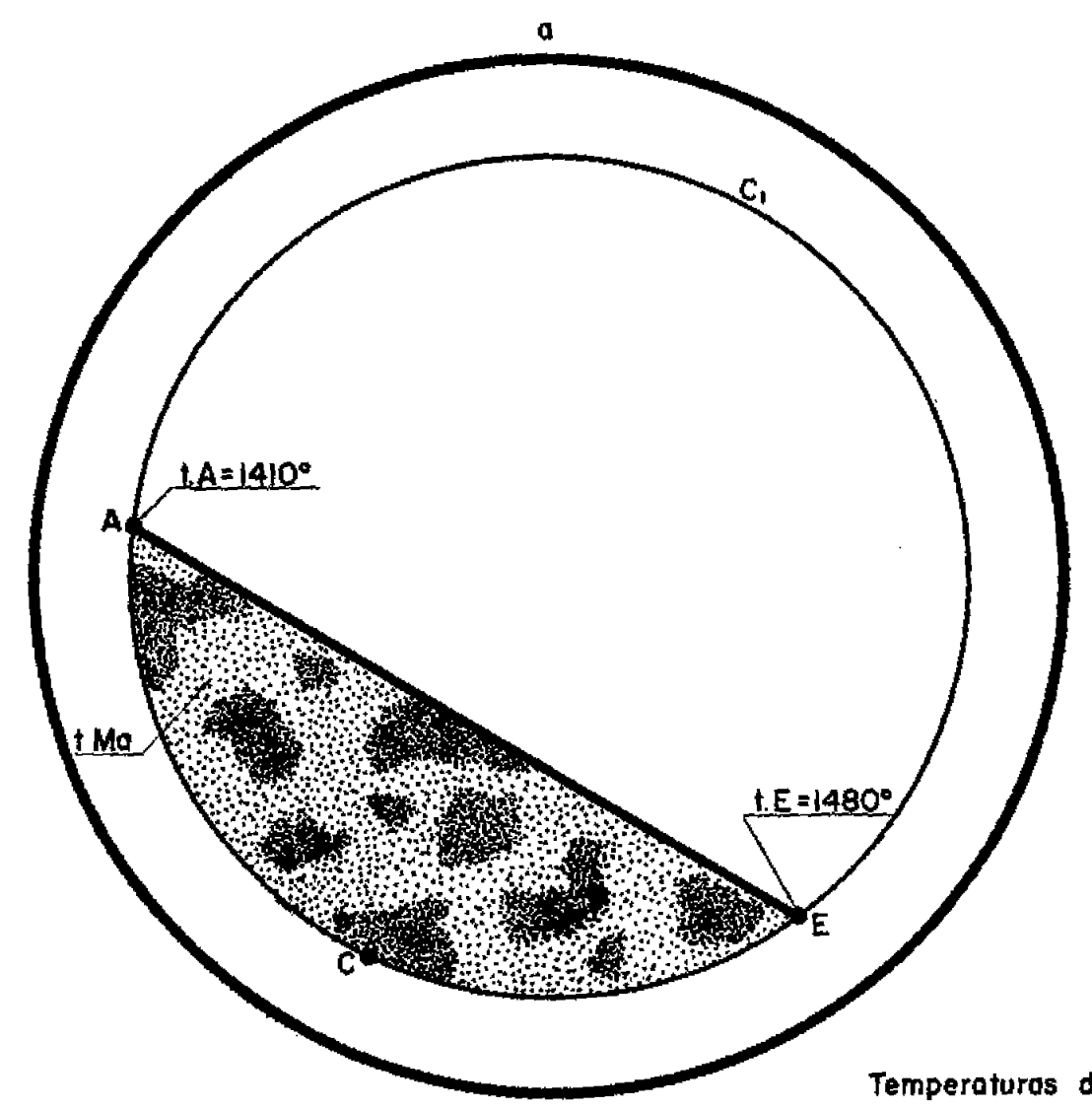

Temperaturas de lo pared para zonos de sinterizacion largas

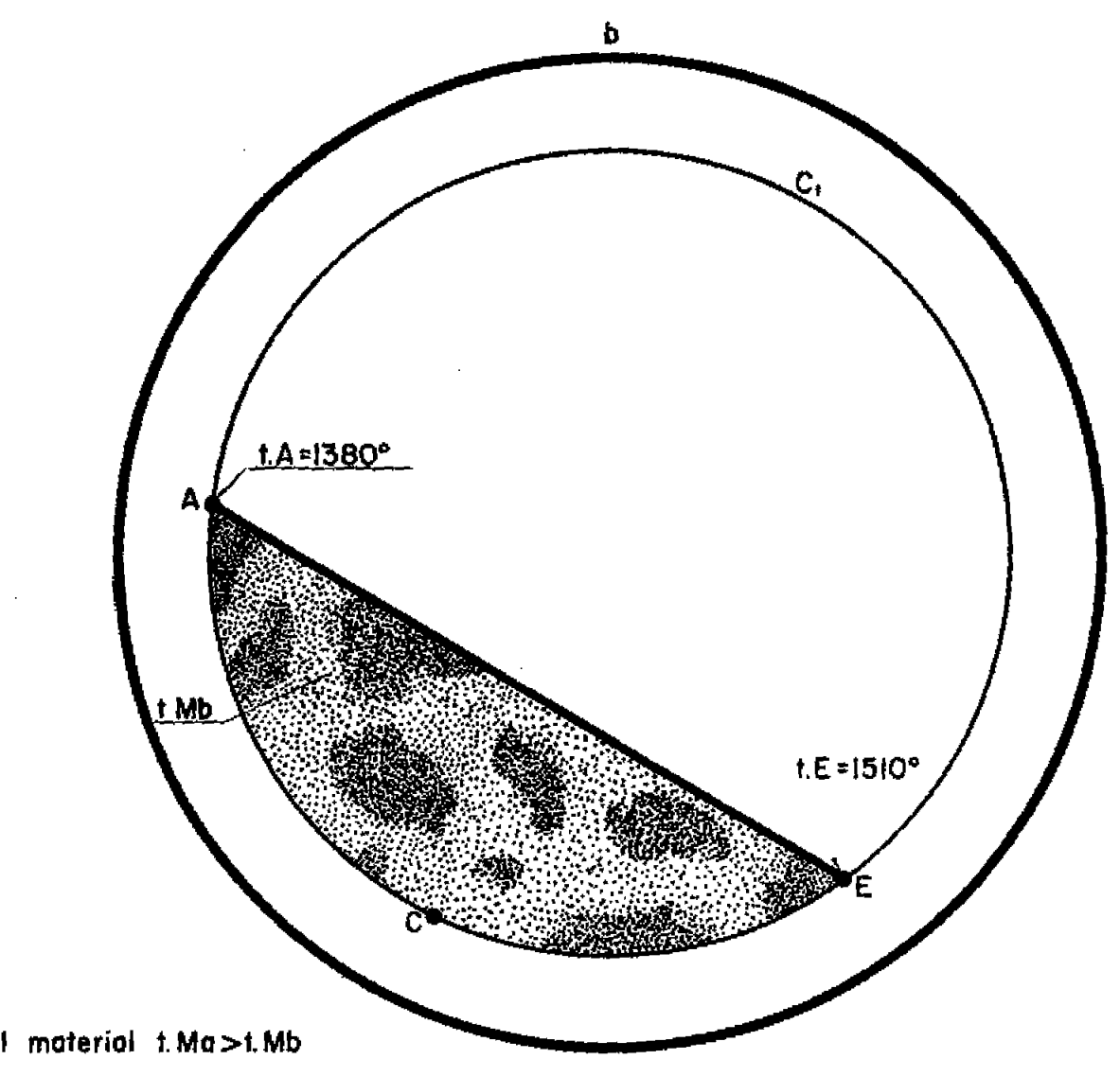

Temperaturas de la pared para
zonos de sinterización.cortas

Fig. ? 


\section{METODOS DE ENSAYO DE LA CORROSION QUIMICA DE REFRACTARIOS}

EN DEFECTO DE MATERIAL DE ATAQUE

EN EXCESO DE material de ataque MEZZCLAS REFRACTARIO
MATERIAL DE ATAQUE

EN DEFECTO DE MATERIAL DE ATAQUE
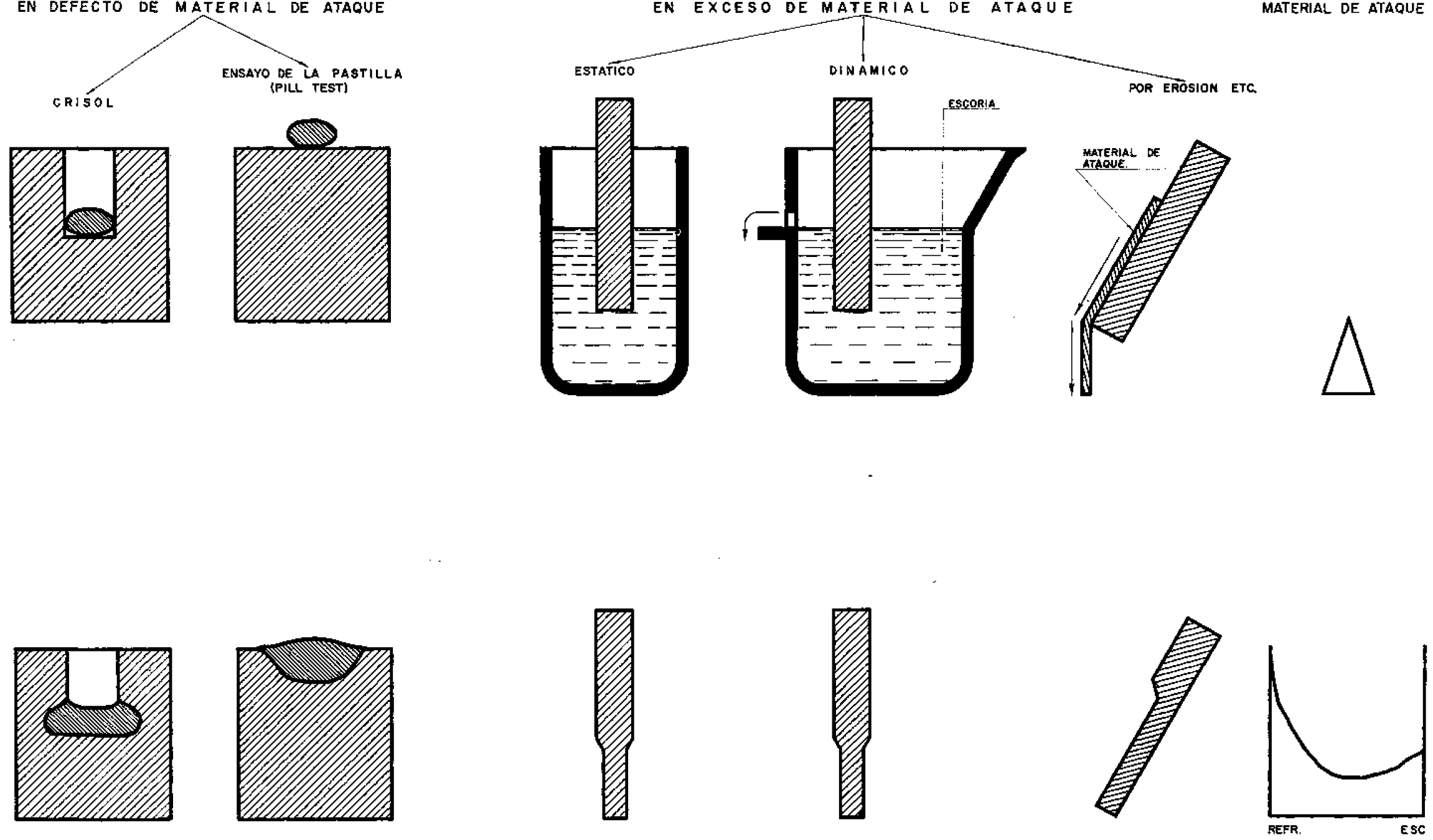

罠

Fig. 8
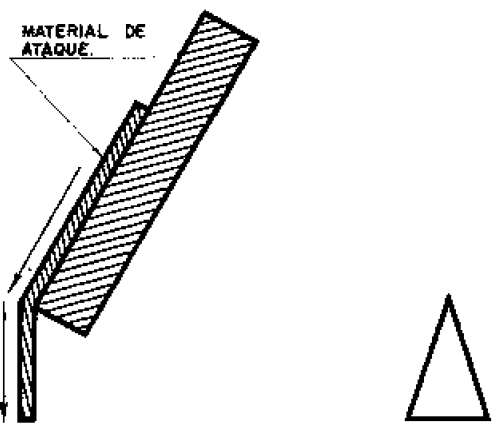
De acuerdo con esto, existe un primer método de enseyo que trata de destacar sólo las características fisicoquímicas del sistema clinker-refractario; consiste en preparar mezclas pulverizadas de refractario $\mathrm{y}$ crudo de cemento en varias proporclones y determinar el punto de fusión o las caracteristicas de reblandecimiento de las mismas.

Fste método permfte, evidentemente, determinar la temperatura minima a la cue el refractario y el clinker forman productos fusibles, pero no da ninguna indicación en cuanto a la influencia de las caracteristicas físicas del refractario en el fenómeno de la corrosíñ.

Los demás métodos de ensayo conocidos, tratan de medir, para un refractario y un material de atague dados, el grado de desgaste del material después de un cierto thempo de tratamiento a una temperatura o temperaturas dades.

Dentro de estos últimos, un primer grupo de métodos tratan de examinar solo el fenómeno de la corroslón, sin ocuparse para neda de la acción mecánica ejercida por el movimiento de la sustancia de ataque (en nuestro caso el clínker), es dectr, conslderando sólo el factor velocidad de reacción refractario-clínker, como resultante de una serie de procesos físicos y quimicos. Entre ellos se encuentran el método del crisol $y$ el ensayo de la pastilla, los cuales tienen lugar en defecto de material de ataque y dan una importancia preponderante a la velocidad de absorción de las fases liquidas.

En este mismo grupo de ensayos tenemos el métodc de inmersión en exceso de escoria fundida, pero suponemos que tiene poco interés en el ceso que nos ocupa.

En un segundo grupo de métodos se encuentran aquellos que consideran de un modo especial las velocidades de aportación y eliminación de materiales en la superficie del ladrillo refractario, los cuales son siempre métodos en exceso de escoria; el de inmersión con circulación y el de pared inclinada con insuflamiento de escoria.

En la figura 8 se reúnen esquemáticamente todos los métodos de ensayo citados anteriormente.

De antemano, es diff́cil fijar cuál es el método más adecuado y menos comparar sus resultados con los que se obtendrian en la práctica industrial; maxime si tenemos en cuenta la variedad de sistemas de hornos existentes. las diferencias de composición quimico-mineralógica de las materias primas y su dosificacion, las de los combustibles empleados $y$, finalmente, ese factor que es el hombre, que tanto puede decidir en la buena marcha de la instalación.

Ígicamente parecen interesantes, aunque sólo sea carcialmente, los ensayos del crisol y de In pastilla, funto con el que estudia las mezclas pulverizadas de ladrillo y clínker. Los primeros, para conocer el comportamiento del ladrillo como unidad estructural frente a la invasión (impregnación) y adherencia de los componentes del clínker, y el segundo, como indice de orientación en la formación y estabilidad de la costra protectora.

En último extremo, los resultados deben complementarse con Ia experiencia del usuario en los distintos materiales y la colaboración de los fabricantes para la preparación de los materiales adecuados a cada caso.

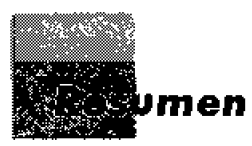

Se mencionan rápidamente las necesidades exigidas por el refractario en un horno de cemento, centrándose, de un modo especial, en la resistencia a Ia corrosion química y Ios factores que la afectan.

Más tarde, se hace un breve recordatorio a Ios diagramas de fases aplicados al sistema refractario-clinker, destacando las precauclones que deben llevarse en su aplicación práctica.

Se expone, más adelante, el posible mecanismo del ataque quimico, extendiéndose, principalmente, en los refractarlos aluminosos, para los que se menclonan los hechos experimentales observados en distintos casos de la práctica industrial. A todo ello se le da una juistificación teórica para confirmar el interés de estos estudios de laboratorio.

Como consecuencia de lo anterior surge la necesidad de una costra protectora, cuyo mecanismo de formacíon se explica con las más diversas opiniones de tipo físico, quimico o físico-quimico.

Finalmente, se exponen los métodos de trabajo de laboratorio que nos pueden dar alguna idea, "a prior"i, del mecanismo de la corrosión quimica del refractario en los hornos de cemento. 


\section{Bibliografia}

5, M. BRISBane y E. R. SEGNIT: "Transactions of the British Ceramic Society", 56, núm, 5, mayo 1957, pág. 237

A. Cocco: "Il Cemento", 55, núm. 10, oct. 1958, pág. 15.

L. Datmad: "Acero y Energia", números 84 (1957), 86 y 90 (1958), y 100 (1960).

J. M." FlRRRR: "Cemento-Hormigon", num. 198, sept. 1950, pág. 266.

C. Goria: "L'Industria Italiana del Cemento", XXIII, num. 2, febrero 1953, póg. 31.

L. HaLM: "Sllicates Industriels", 17, núm, 3, marzo 1952, pág. 77.

L. HaLm: "Documentation métallurgique de la Société de l'Industrie Minérale", núm. 3, julio-agosto-septiembre, 1951

F. P. Hall y H. INSLeY: "Phase Diagrams for Ceramists". American Ceramic Society, 1947, Columbus (Ohí).

R. HAJEnschikLD: “Zement”, 31, 1942, pág. 225.

L. G. Hvegert: "Transactions of the British Ceramic Scciety", 56, núm. 3, marzo 1957, pag. 87.

J. JAMaIS: "Silicates Industriels", XXVI, núm. 1, enero 1961, pág. 25.

K. KonoptcкY: "Zement-Kalk-Gips", 4, núm. 9, sept. 1951, pág. 240.

F. M. I,EA y T. W. PARKGr: "Butlding Research station, Technical Paper", núm. 16, 1935.

K. LkRcrr: "Ber. Deut. Keram. Ges.", 25, 1944, pág. 1.

G. Mussanda: "Materlales, Maquinaria y Métodos para la Construcción", 1, nủm. 4, marzo 1957, pág. 3.

J. A. PAsK: Comunicación privada, enero 1961.

J. R. Rarr: "Basic Refractories", Iliffe and Sons, Ltda., 1960, London.

F. SorIs: "Informes de la Construcción", núm. 63 ,agosto-septiembre 1954.

F. Sorm: Instituto Eduardo Torroja de la Construcción y del Cemento, Monografía núm. 165.

K. Spangenberg: "Zement-Kalk-Gips", 4, diciembre 1951, pág. 317, 